\title{
Microlensing towards LMC and M31
}

\author{
Ph. Jetzer ${ }^{1}$, A. Milsztajn ${ }^{2}$ and P. Tisserand ${ }^{2}$ \\ ${ }^{1}$ Institute of Theoretical Physics, University of Zürich, Winterthurerstasse 190, CH-8057 \\ Zürich, Switzerland, email: jetzer@physik.unizh.ch \\ ${ }^{2}$ Dapnia, Service de Physique des Particules, CEA-Saclay, F-91191 \\ Gif-sur-Yvette, France; email: amilsztajn@cea.fr, ptisserand@cea.fr
}

\begin{abstract}
The nature and the location of the lenses discovered in the microlensing surveys done so far towards the LMC remain unclear.

This contribution is comprised of two distinct parts. In the first part, motivated by these questions, we compute the optical depth for the different intervening populations and the number of expected events for self-lensing, using a recently drawn coherent picture of the geometrical structure and dynamics of the LMC disk. By comparing the theoretical quantities with the values of the observed events it is possible to put some constraints on the location and the nature of the machos. Clearly, given the large uncertainties and the few events at our disposal it is not yet possible to draw sharp conclusions, nevertheless we find that up to 3-4 macho events might be due to lenses in LMC, which are most probably low mass stars, but that hardly all events can be due to self-lensing. A plausible solution is that the events observed so far are due to lenses belonging to different intervening populations: low mass stars in the LMC, in the thick disk, in the spheroid and some true machos in the halo of the Milky Way and the LMC itself. We report also on recent results of microlensing searches in direction of the M31 galaxy, by using the pixel method. The present analysis still does not allow yet to draw sharp conclusions on the macho content of the M31 galaxy.

In the second part (section 5), a preliminary account of the final results from the EROS-2 programme is presented. Based on the analysis of 33 million LMC and SMC stars followed during 6.7 years, strict limits on the macho content of the galactic halo are presented; they cover the range of macho masses between 0.0001 and 100 solar mass. The limits are better than $20 \%$ (resp. $5 \%$ ) of the standard halo for masses between 0.0002 and 10 (resp. 0.001 to 0.1 ) solar mass. This is presently the data set with the largest sensitivity to halo machos.
\end{abstract}

\section{Introduction}

Since Paczyński's original proposal (Paczyński 1986) gravitational microlensing has been proven to be a powerful tool for the detection of the dark matter component in galactic haloes in the form of machos. Searches in our Galaxy towards the LMC show that up to $20 \%$ of the halo could be formed by objects of around $M \sim 0.4 M_{\odot}$.

However, the location and the nature of the microlensing events found so far towards the LMC is still a matter of controversy. The MACHO collaboration found 13 to 17 events in 5.7 years of observations, with a mass for the lenses estimated to be in the range $0.15-0.9 M_{\odot}$ assuming a standard spherical Galactic halo (Alcock et al. (2000a)). From this, they derived a total optical depth towards the LMC of $\tau=1.2_{-0.3}^{+0.4} \times 10^{-7}$. The EROS2 collaboration has announced at this symposium the detection of 4 candidate events (one of which being most probably due to a lens located in the disk of our Galaxy) based on 6.7 years of observation but monitoring about three times as much stars as the MACHO collaboration. These EROS2 results are detailed in section 5 of this contribution. The MACHO collaboration monitored primarily the central part of the LMC, whereas 
the EROS2 experiment covered a larger solid angle but in less crowded fields. The EROS2 microlensing rate should thus be less affected by self-lensing. This might be the reason for the fewer events seen by EROS2.

The analysis of Jetzer et al. (2002) and Mancini et al. (2004) has shown that probably the observed events are distributed among different galactic components (disk, spheroid, galactic halo, LMC halo and self-lensing). This means that the lenses do not belong all to the same population and their astrophysical features can differ deeply.

Some of the events found by the MACHO team are most probably due to self-lensing: the event MACHO-LMC-9 is a double lens with caustic crossing (Alcock et al. 2000b) and its proper motion is very low, thus favouring an interpretation as a double lens within the LMC. The source star for the event MACHO-LMC-14 is double and this has allowed to conclude that the lens is most probably in the LMC. The event LMC-5 is due to a disk lens and indeed the lens has even been observed with the HST, see Alcock et al. (2001) The other stars which have been microlensed were also observed but no lens could be detected, thus implying that the lens cannot be a disk star but has to be either a true halo object or a faint star or brown dwarf in the LMC itself.

Thus up to now the question of the location of the observed MACHO events is unsolved and still subject to discussion. Clearly, with much more events at disposal one might solve this problem by looking for instance at their spatial distribution. To this end a correct knowledge of the structure and dynamics of the luminous part of the LMC is essential, and we take advantage of a new picture of the LMC disk.

Searches towards M31 have also been proposed (Crotts (1992), Baillon et al. (1993) Jetzer (1994)). This allows to probe a different line of sight in our Galaxy, to globally test the M31 halo and, furthermore, the high inclination of the M31 disk is expected to provide a strong signature (spatial distribution) for halo microlensing signals. For extragalactic targets, due to the distance, the sources for microlensing signals are not resolved. This calls for an original technique, the pixel method, the detection of flux variations of unresolved sources, the main point being that one follows flux variations of every pixel in the image instead of single stars. Several collaborations have recently presented a handful of microlensing events (SLOTT-AGAPE Calchi Novati et al. (2003), POINT-AGAPE Paulin-Henriksson et al. (2003). WeCapp Riffeser et al. (2003) and MEGA de Jong et al. (2004) .

\section{LMC optical depth}

In a series of interesting papers (van der Marel et al. (2001) van der Marel et al. (2002)), a new coherent picture of the geometrical structure and dynamics of the LMC has been given. In the following analysis we use this model and adopt the same coordinate system and notations as in van der Marel et al. The center of the disk coincides with the center of the bar and its distance from us is $D_{0}=50.1 \pm 2.5 \mathrm{kpc}$. We take a bar mass $M_{\text {bar }}=1 / 5 M_{\text {disk }}$ with $M_{\text {bar }}+M_{\text {disk }}=M_{\text {vis }}=2.7 \times 10^{9} \mathrm{M}_{\odot}$.

We consider also the LMC halo contribution to the optical depth. We use two different models to describe the halo density profile: a spherical halo and an ellipsoidal halo. The values of the parameters have been chosen so that the models have roughly the same mass within the same radius. For the spherical model we adopt a classical pseudo-isothermal spherical density profile, with a halo mass within a radius of $8.9 \mathrm{kpc}$ equal to $5.5 \times 10^{9} \mathrm{M}_{\odot}$.

The computation of the optical depth is made following the method outlined in Jetzer et al. (2002)

In Fig. 11 we report the optical depth contour map for self-lensing, i.e. for events where both the sources and the lenses belong to the disk and/or to the bulge of LMC. As expected, there is almost no near-far asymmetry and the maximum value of the optical depth, $\tau_{\max } \simeq 4.80 \times 10^{-8}$, is reached in the center of LMC. The optical depth then 


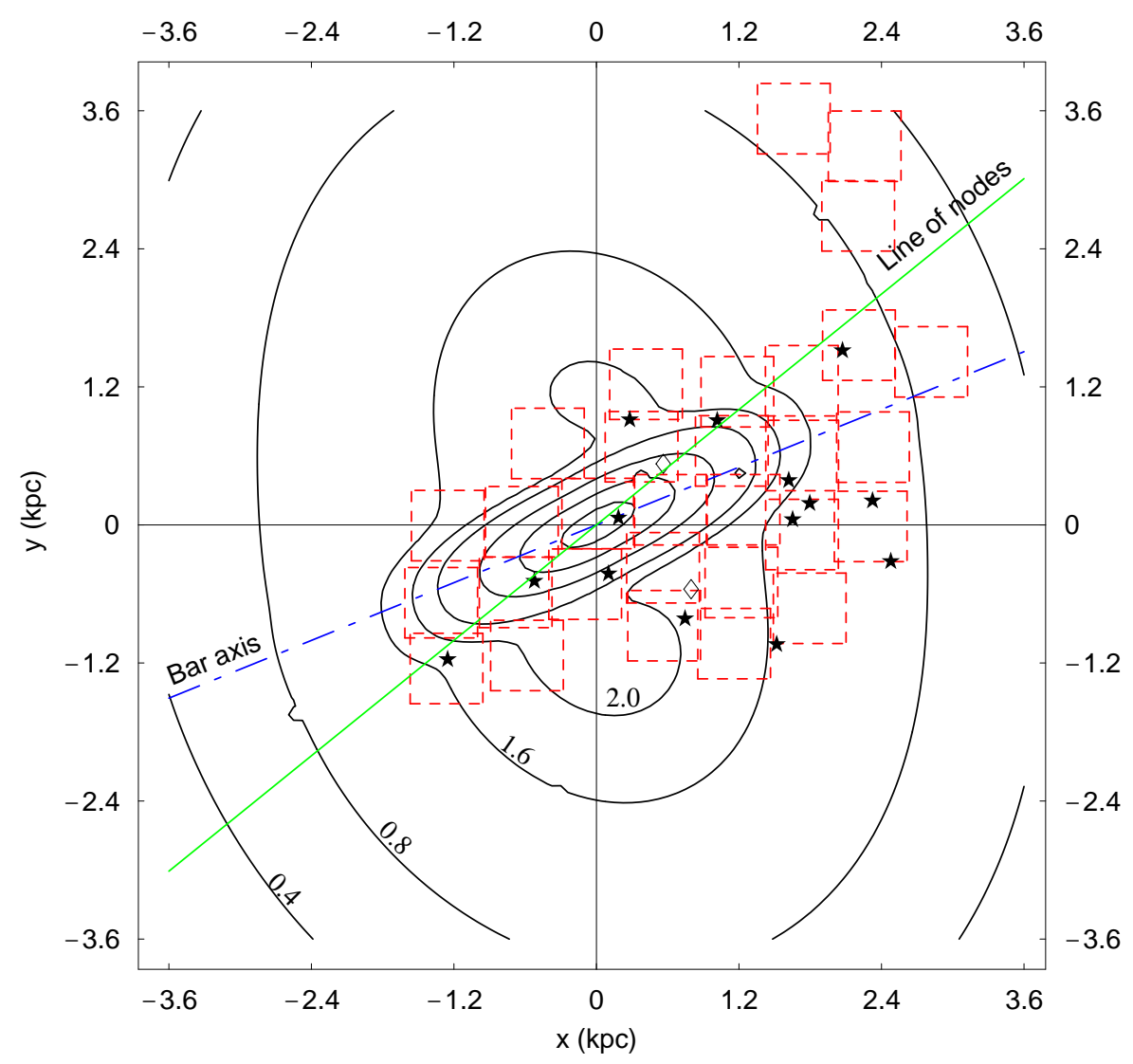

Figure 1. Contour map of the optical depth for self-lensing. The locations of the MACHO fields and of their microlensing candidates are also shown. The numerical values are in $10^{-8}$ units. The innermost contours correspond to values $2.4 \times 10^{-8}, 3.2 \times 10^{-8}, 4.0 \times 10^{-8}$ and $4.6 \times 10^{-8}$ respectively.

rapidly decreases, when moving, for instance, along a line going through the center and perpendicular to the minor axis of the elliptical disk, that coincides also with the major axis of the bar. In a range of about only $0.80 \mathrm{kpc}$ the optical depth quickly falls to $\tau \simeq 2 \times 10^{-8}$, and afterwards it decreases slowly to lower values. We computed also the optical depth contour maps for lenses belonging to the halo of LMC in the case of spherical model in the hypothesis that all the LMC dark halo consists of compact lenses. The ellipsoidal model leads to similar results (Mancini et al. (2004)). A striking feature of the map is the strong near-far asymmetry, which is not expected, on the contrary, for a self-lensing population of events.

\section{LMC self-lensing event rate}

We evaluated the microlensing rate in the self-lensing configuration, i.e. lenses and sources both in the disk and/or in the bar of LMC. We have taken into account the transverse motion of the Sun and of the source stars. We assumed that, to an observer 


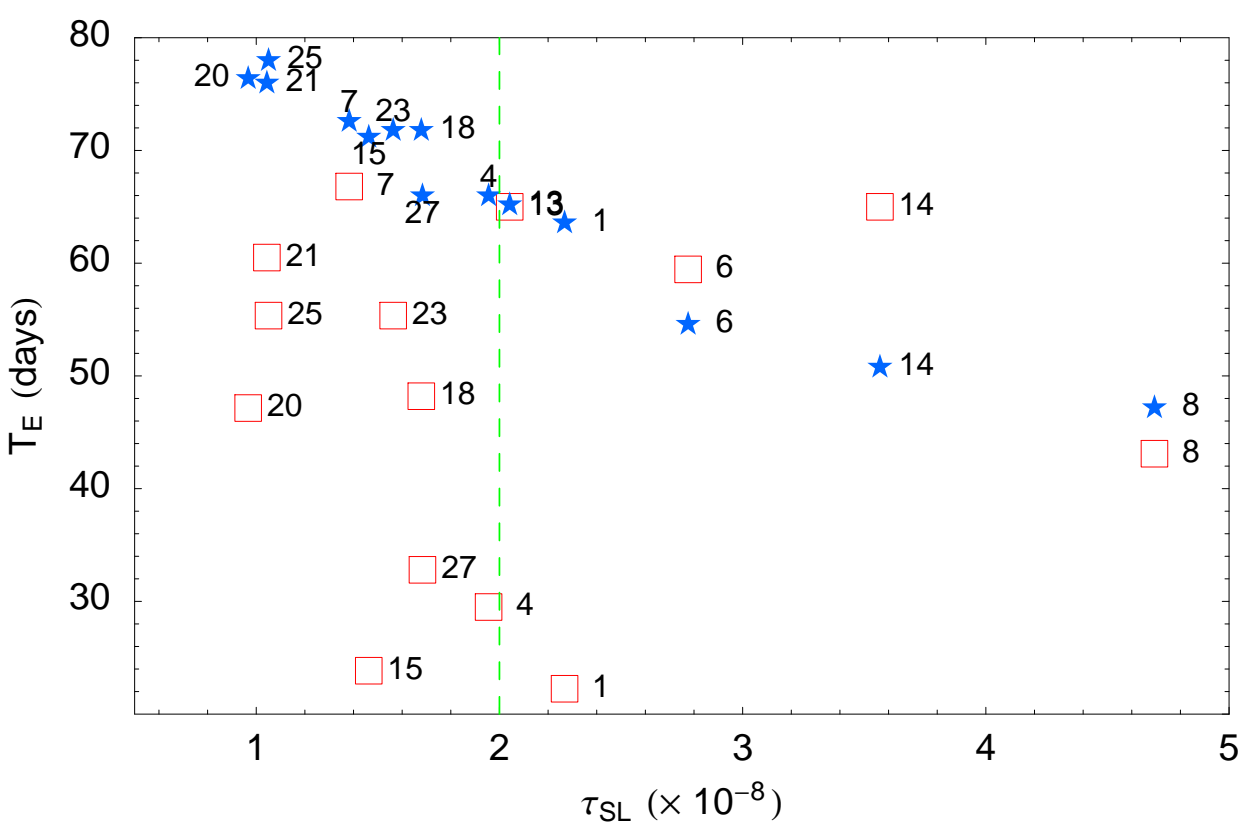

Figure 2. Scatter plot of the observed (empty boxes) values of the Einstein time and of the expected values of the median $T_{\mathrm{E}, 50 \%}$ (filled stars), with respect to the self-lensing optical depth evaluated along the directions of the events.

comoving with the LMC center, the velocity distributions of the source stars and lenses have a Maxwellian profile, with spherical symmetry.

In the picture of van der Marel et al., within a distance of about $3 \mathrm{kpc}$ from the center of LMC, the velocity dispersion (evaluated for carbon stars) along the line of sight can be considered constant, $\sigma_{\text {los }}=20.2 \pm 0.5 \mathrm{~km} / \mathrm{s}$. Summing over all fields we find that the expected total number of self-lensing events is $\sim 1.2$. Clearly, taking also into account the uncertainties in the parameter used following the van der Marel model for the LMC the actual number could also be somewhat higher but hardly more than our upper limit estimate of about 3-4 events given in Jetzer et al. (2002)

As a further argument, assuming all the 14 events as self-lensing, we study the scatter plots correlating the self-lensing expected values of some meaningful microlensing variables with the measured Einstein time or with the self-lensing optical depth. In this way we can show that a large subset of events is incompatible with the self-lensing hypothesis.

In Fig. 2] we report on the $y$-axis the observed values of the Einstein time $T_{\mathrm{E}}$ (empty boxes) as well as the expected values for self-lensing of the median $T_{\mathrm{E}, 50 \%}$ (filled stars) evaluated along the directions of the events. On the $x$-axis we report the value of the selflensing optical depth calculated towards the event position; the optical depth is growing going from the outer regions towards the center of LMC according to the contour lines shown in Fig. 1. An interesting feature emerging clearly is the decreasing trend of the expected values of the median $T_{\mathrm{E}, 50 \%}$, going from the outside fields with low values of $\tau_{\mathrm{SL}}$ towards the central fields with higher values of $\tau_{\mathrm{SL}}$. The variation of the stellar number density and the flaring of the LMC disk certainly contributes to explain this behaviour.

We now tentatively identify two subsets of events: the nine falling outside the contour

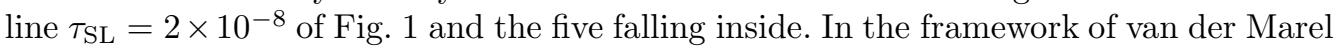
et al. LMC geometry, this contour line includes almost fully the LMC bar and two ear 
shaped inner regions of the disk, where we expect self-lensing events to be located with higher probability.

This plot suggests that the self-lensing events have to be searched among the cluster of events with $\tau_{\mathrm{SL}}>2 \times 10^{-8}$, and at the same time that the cluster of the 9 events including LMC-1 belongs, very probably, to a different population.

Moreover, when looking at the spatial distribution of the events one sees a clear nearfar asymmetry in the van der Marel et al. geometry; they are concentrated along the extension of the bar and in the south-west side of LMC. Indeed, we have performed a statistical analysis of the spatial distribution of the events, which clearly shows that the observed asymmetry is greater than the one expected on the basis of the observational strategy (Mancini et al. (2004)).

Note that, since this study was completed, event MACHO-LMC-23 displayed a new variation in the EROS2 data (see section 5.4 and Fig. 4).

\section{Pixel lensing towards M31}

The SLOTT-AGAPE collaboration has been using data collected on the 1.3m McGrawHill Telescope at the MDM observatory, Kitt Peak (USA). Observations have been carried out in a two years campaign, from October 1998 to the end of December 1999. As a final result of the analysis, taking into account also an extension of the light curve using INT data, gives three possible candidate events one of which is reported in Fig. 3

The POINT-AGAPE collaboration carried out a survey of M31 by using the Wide Field Camera (WFC) on the $2.5 \mathrm{~m}$ INT telescope. A first analysis (Paulin-Henriksson et al. (2003)) was made with the aim to detect short $\left(t_{1 / 2}<25\right.$ days $)$ and bright variations $(\Delta R<21$ at maximum amplification), compatible with a Paczyński signal. The first requirement is suggested by the results on the predicted characteristics of microlensing events of a Monte Carlo simulation of the experiment. As an outcome, seven light curves are detected (Paulin-Henriksson \& Calchi Novati (2004)) which can be considered viable microlensing events.

Once a microlensing event is detected it is important, given the aim to probe the halo content in form of macho, to find out its origin, namely, whether it is due to self-lensing within M31 or to a macho. This is not straightforward. The spatial distribution of the events is an important tool, but still unusable given the small statistics.

The light curve of the event PA-99-N2 is particularly interesting because it shows clear deviations from a Paczyński shape, while remaining achromatic (and unique) as expected for a microlensing event. After exploring (An et al. (2004) ) different explanations, it is found that the observations are consistent with an unresolved RGB or AGB star in M31 being microlensed by a binary lens, with a mass ratio of $\sim 1.2 \times 10^{-2}$.

\section{Results from the final EROS-2 data set}

In a first phase (1990-95), the EROS group (Expérience de Recherche d'Objets Sombres) conducted a search for low mass machos (where no candidate were found in the mass range $10^{-7}$ to $10^{-3}$ solar mass), and a medium sensitivity search to higher mass objects $\left(10^{-4}\right.$ to 1 solar mass). The latter was designed to find stellar mass machos, should they be the sole constituent of the galactic halo. Only two candidates were found, where seven were expected. Results from this first phase can be found in Renault et al. (1997) and references therein.

In order to better study this possible signal, a second survey of the Magellanic Clouds was started (EROS-2), with the aim of improving the sensitivity by at least an order of 

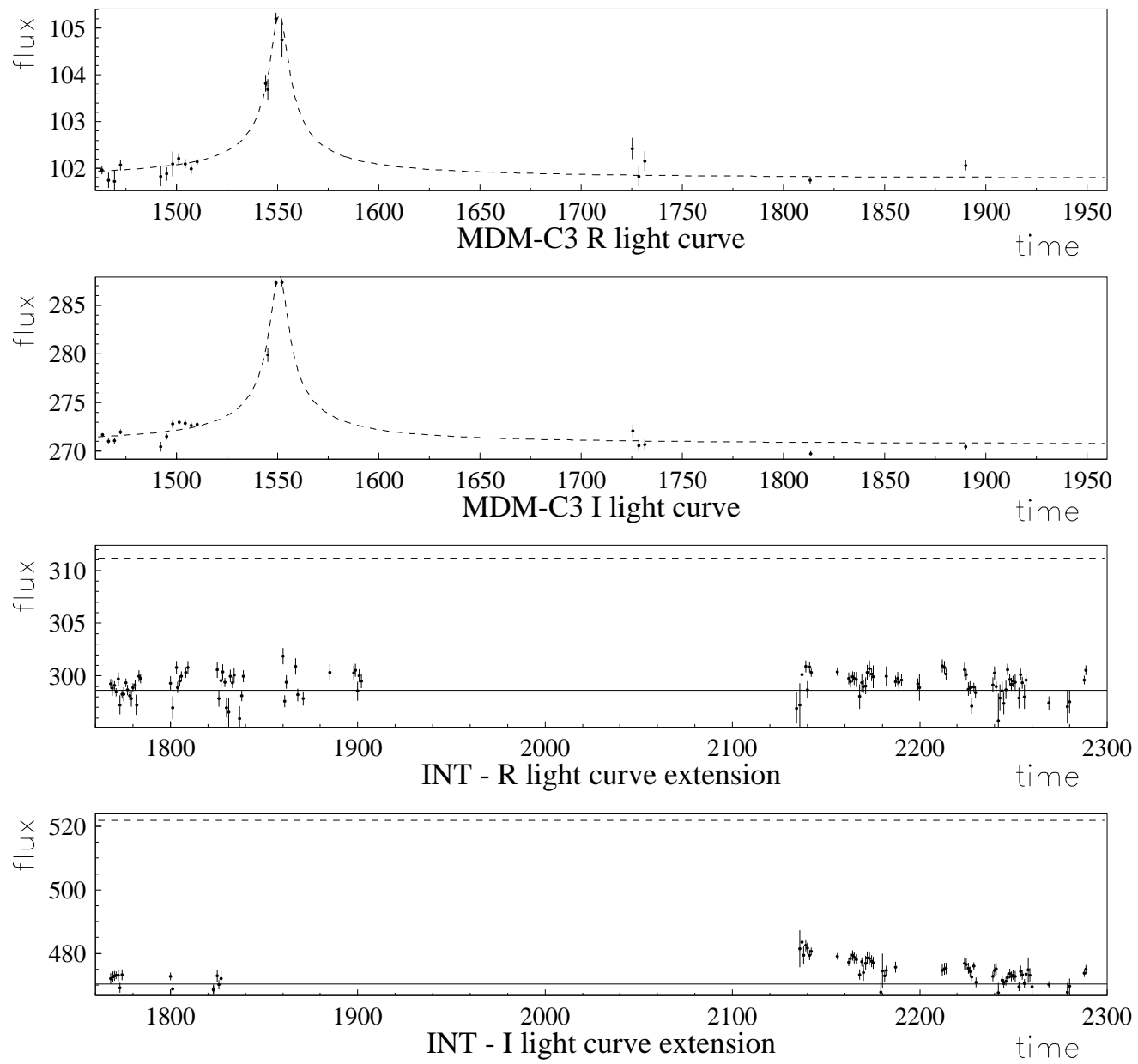

Figure 3. MDM C3 light curves together with their extension into the INT data. On the $y$ axis, flux is in ADU/s; on the $x$ axis, time is in days, with the origin in J-2449624.5 (both data sets). For the MDM light curves the dashed line represent the result of the Paczyński fit. For the INT light curves, shown together with the solid line representing the baseline is a dashed line representing the level of the maximum deviation of flux found on the corresponding MDM light curve.

magnitude. It was conducted between July 1996 and February 2003, using a 1 m dedicated telescope, the MARLY, and two wide field 32 million pixel CCD mosaics covering $1 \mathrm{deg}^{2}$. Details of the setup and operations can be found in Tisserand (2004) and references therein. We present here the first, preliminary account of final results using the full Magellanic Clouds data set of EROS-2. Interim reports on part of the data were presented in Lasserre et al. (2000) and Afonso et al. (2003)

The total number of stars followed was 55 million (including 6 in the SMC), compared to 4 million LMC stars in the EROS-1 phase. The monitored solid angle is 88 (10) deg ${ }^{2}$ in the LMC (SMC). (For comparison, the MACHO collaboration monitored 11 million stars over $15 \mathrm{deg}^{2}$, mostly in the central region of the LMC (Alcock et al. (2000a)). We decided to restrict our analysis to the 33 million brightest stars in our sample (29 in the LMC, 4 in the SMC). In order to analyse a homogeneous sample, all Magellanic Clouds images were photometered anew, using better and slightly deeper template images than 
in our previous reports. The typical sampling of the light curves is about twice per week in each of two passbands.

We have conducted two independent analyses, aimed at different macho timescales. When the Einstein timescale is shorter than about two years, the microlensing light curves display a visible baseline flux. The first analysis relies on identifying this baseline, and departures from it. For longer timescales, because of the six-year duration of the EROS-2 programme, the baseline is not readily seen (or even not at all). The second analysis thus relies on light curve shape criteria.

\subsection{Shorter timescale events and their backgrounds}

In the first analysis, we require a single excursion from the baseline, that is regular and seen in both passbands; at this stage, it should loosely agree with the expected microlensing light curve shape. The selected sample contains mostly variable stars, but also unphysical variations that originate in PSF variations corresponding to optical maintenance of our setup. We characterize and eliminate this spurious signal. The variable stars come mainly in three categories : "blue bumpers", novae and supernovae. Blue bumpers are upper main sequence stars that display limited variations (less than $70 \%$ ), that are chromatic (larger in the red passband). This chromaticity cannot be explained by blending in a microlensing phenomenon. Specific cuts deal with this background. Novae and supernovae have asymmetric light curves, with a faster rise time. Magellanic Clouds novae are easily identified and rejected : they are very bright objects with very asymmetric light curves.

After rejection of blue bumpers and novae, supernovae in galaxies behind the LMC and SMC represent the dominant population in our sample of microlensing candidates at the end of the analysis. We find 28 such objects, a rate comparable to that found by MACHO, see Alcock et al. (2000a) Most of these occur in outer regions of the LMC. Supernovae are rejected by performing a fit of a function that includes an asymmetry parameter $S$, and reduces to the usual symmetric microlensing light curve when $S=0$, see Tisserand (2004) The function is obtained by replacing in the usual microlensing formula the Einstein timescale $t_{E}$ with $t_{E}\left[1+S \arctan \left(\left(t-t_{0}\right) / t_{E}\right)\right]$. We reject all candidates that have a large asymmetry parameter, $|S|>0.3$. In addition, the neighbourhood of all microlensing candidates is inspected visually on the template images. For asymmetric light curves, in more than half of the cases, a galaxy or even a cluster of galaxies is visible near the candidate (less than $30 \mathrm{arcsec}$ ). The probability of a chance alignment is very low. This reinforces their interpretation as distant supernovae; from their flux, we estimate their distance between 500 and $1000 \mathrm{Mpc}$.

\subsection{SMC results}

The analysis of the SMC data has selected two events. One has been known already since 1997, see Alcock et al. (1997b), Palanque-Delabrouille et al. (1998), The other one is situated in between the main sequence and the red giant clump, in a thinly populated domain of the colour-magnitude diagram. The publically available MACHO group data on this object display further variation, allowing to reject it. (Note that this star lies in the same domain as candidate EROS1-LMC-1, which has been shown to be a Be variable star, see the discussion below). Three long duration candidates had been detected in the analysis by Afonso et al. (2003) of 5 years of EROS-2 SMC data; they did not agree well with a microlensing shape and were thus considered dubious. They now display further variability in the additional 2 years available here; they have not been selected by our criteria.

The single EROS-2 microlensing candidate towards the SMC has a long timescale, 
125 days. If due to a halo microlens, its light curve should display a "microlensing parallax", i.e. a deformation due to the Earth's motion around the Sun. Such a deformation was detected neither in the EROS-2 data, nor in the MACHO data. From this, it can be concluded that, at $97 \%$ C.L., the lensing object lies in the SMC. Thus, the optical depth corresponding to this event $\left(710^{-8}\right)$ is probably best explained by the so-called SMC "self-lensing".

\subsection{LMC results}

The present analysis of the LMC data has selected four new candidates. (The status of the former EROS LMC microlensing candidates is discussed in the next section.) One of the candidates, EROS2-LMC-8, is a high signal-to-noise microlensing light curve. It shows a chromatic variation of a factor $12(25)$ in the red (visible) band. Its position in the colour-magnitude diagram is abnormal, almost 1 mag redder than comparable objects in the main sequence. When a possible blending is taken into account however, the microlensing fit becomes excellent, with agreement between the timescales in the two bandpasses. The baseline flux corresponding to the magnified object is within the dimmer part of the observed LMC main sequence (22.5); this is compatible with the source being an LMC star. In contrast, the unmagnified flux is now even redder, and cannot correspond to an LMC star.

We have investigated whether the unmagnified flux could correspond to the lens being a $\operatorname{dim} \mathrm{M}$ dwarf in the galactic disk, much in the same way as for event MACHO-LMC-5, see Alcock et al. (2001). We find a good solution for a 13.5 mag M dwarf (absolute mag) at a distance of $300 \mathrm{pc}$. A simplified Monte-Carlo simulation shows that the expected median distance of a disk lens is close to $700 \mathrm{pc}$, in reasonable agreement. The observed timescale then corresponds to a transverse velocity of $50 \mathrm{~km} / \mathrm{s}$, again a normal value. The median expected amplification for such a lens-source configuration is found to be 25 , and the observed value is 40 . Our interpretation of this event could be strengthened by better angular resolution images of the source, similarly to Alcock et al. (2001) We expect, at the time of this symposium, that the lens-source separation is 0.15 arcsec.

The three other candidates, EROS2-LMC-9 to 11, have lower signal-to-noise. The source stars have a baseline flux at about 21st mag and the timescales range from 35 to 55 days. The magnifications are close to a factor three. Candidate 10 is in an abnormal region of the colour-magnitude diagram, 0.5 mag redder than comparable objects in the main sequence. It shows the most chromatic variation of these three candidates, and its rise time $(20 \mathrm{~d})$ is shorter than its decrease time. For these reasons, we suspect it may be another distant supernova that our cuts were not strict enough to reject, possibly the dimmest SN in our analysis. Investigation of this candidate is going on.

The analysis described here basically looks for regular variations, such as those of simple (point source, point lens) microlensing. It is also able to detect microlensing phenomena with minor deviations from this simple case, such as "parallax" deformations or source size effects. It may not however be sensitive to caustic-crossing double lens events, such as MACHO-SMC-98-2, see e.g. Afonso et al. (2000) For that reason, we have performed another analysis that looks for stars with very fast variations, of at least 0.5 mag per day, a typical value in caustic crossing events. This analysis selected only novae in the Magellanic Clouds. No caustic crossing candidate was found.

\subsection{Follow-up of the published microlensing candidates}

Former LMC microlensing candidates have already been retracted after having been observed for a longer period of time. They had displayed a second variation, that was very unlikely to be due to microlensing of the second, widely separated component of 
a double source star; the most plausible explanation seemed intrinsic stellar variability rather than microlensing.

These retracted candidates are: EROS1-LMC-2, presented in Aubourg et al. (1993) and which displayed a new variation 8 years later, see Lasserre et al. (2000) MACHOLMC-2 and 3, presented in Alcock et al. (1995) and retracted in Alcock et al. (1997a) and candidate EROS2-LMC-4, presented in Lasserre et al. (2000) and retracted in Milsztajn \& Lasserre (2001) The fraction of retracted candidates is small, but non negligible, and no one knows how it will evolve if all candidates are followed for a much longer period of time. We have used our new data set to perform a follow-up of all published microlensing candidates towards the Magellanic Clouds, both by EROS and by MACHO. The final EROS2 data provides an additional 6.6, 3.7 and 5.1 years time base compared to the latest publications by EROS1, Aubourg et al. (1993), EROS2, Milsztajn \& Lasserre (2001) and MACHO, Alcock et al. (2000a), to check for the stability of the baseline.

There were 5 surviving microlensing candidates from EROS, one from EROS1 (number 1) and four from EROS2 (numbered 3, 5, 6 and 7). Candidate 1 displayed a new variation in 1998, 6.3 years after the first one, of similar amplitude (a factor two) and timescale $(28 \mathrm{~d})$. This second variation is well fit by a microlensing light curve. Because they are separated in time by more than 80 Einstein timescales, the probability that these two bumps correspond to the microlensing of a double source star is lower than half a percent. This candidate is thus rejected. Moreover, it was already known to be a Be star (Beaulieu et al. (1995)) and was thus suspected of being variable. Candidate 3 also displayed new, irregular variations between 1999 and 2002, and was thus rejected.

The light curves of candidates 5 to 7 have been improved, due to the better template images in the present analysis. This made apparent an asymmetry in rise and fall times, that allowed to identify them as supernovae. Note that there were no cut targeted at rejecting distant supernovae in previous EROS analyses, and that our candidate 5 is identical to MACHO candidate 26, which was rejected by Alcock et al. (2000a) for the very same reason.

The conclusion is that none of the former EROS microlensing candidates are still considered valid.

We have also attempted to follow all $13 \mathrm{MACHO}$ candidates selected by their analysis A (stricter cuts). We were able to identify unambiguously nine of them. Our data provide 5.1 (resp. 3.1) additional years compared to the published (resp. Web available) light curves. One star, MACHO-LMC-23, displays a new variation in Dec 2001, very similar to that observed by the MACHO group in Feb 1995 (variation of 1 mag and timescale of 40 days). The corresponding light curve is shown in Fig. [ Note that this variation is compatible with being achromatic, and is also well fit by a microlensing light curve.

Figure 4. The light curves of candidate MACHO-LMC-23, as seen by EROS2 between 1996 and 2003 (top : red passband; bottom : visible). The flux is in arbitrary units; the time is in days, with the origin at JD $=2450000$. The bump at day 2250 is a new variation, 6.8 years after that originally seen by the MACHO group, before the startup of EROS2.

\subsection{Limits on the macho content of the halo}

This search for microlensing phenomena with timescales shorter than 2 years in the complete EROS2 Magellanic Clouds data set has selected four LMC candidates and one SMC candidate. The two high signal-to-noise events are most likely microlensing due to an SMC lens (the SMC candidate) and a galactic disk lens (event 8 towards the LMC). The remaining 3 events, if interpreted as machos, correspond to an optical depth of $1.510^{-8}$, i.e. $3 \%$ of the standard spherical halo. Taking a more conservative approach, 
one can choose to present a 95\% C.L. upper limit on the macho content of the halo, based on these three candidates. A preliminary version of this limit is shown in Fig. I It uses only the EROS2 LMC data set; combination with both the EROS1 and the EROS2 SMC results will be done later. The limit is better than $12 \%$ of the halo for macho masses between 0.0002 and 1 solar mass.

This limit is compared to the previous EROS2 LMC result of Lasserre et al. (2000) to show the increase in sensitivity since 2000. It is also compared to the signal presented by Alcock et al. (2000a) Our result is compatible with the lowest part of the domain allowed by the MACHO group analysis. It must be recalled however that the fields monitored by MACHO and EROS2 are not identical. A better comparison is in progress; in particular, we are trying to evaluate whether the EROS2 result is compatible with the central value of Alcock et al. (2000a), i.e. a $20 \%$ macho component of the halo.

Figure 5. The preliminary 95\% C.L. upper limit on the macho content of the halo obtained from the final EROS2 LMC data only (red curve between 0.0001 and 100 solar masses). The lower cross is the value of the optical depth towards the LMC corresponding to the three candidates. The dashed line that goes through this cross shows what the limit would be in the absence of microlensing candidates. This limit is compared to that presented in Lasserre et al. (2000) using the same method, but all EROS data sets available at the time (green curve between $10^{-7}$ and 10 solar mass). The smaller orange-shaded contour between 0.1 and 1 solar mass is the signal presented by Alcock et al. (2000a) with the lighter cross indicating their preferred solution.

Finally, we remark that the present value of the LMC optical depth from EROS2 seems to be compatible with "self-lensing" within the LMC.

\subsection{Longer timescale events}

We have also searched for events with timescales longer than two years in the EROS2 data, using only light curve shape criteria. This analysis is still in progress. At present, 10 candidates have been selected. All but one are located in the central fields of the LMC, that contain $30 \%$ of the stars monitored by EROS2. This in itself shows that they cannot all be microlensing phenomena caused by halo objects, as such events should follow the LMC stellar distribution. No candidate was selected towards the SMC.

Nine of these 10 events are found in the MACHO publically available light curve database. The combination of the MACHO and EROS2 data indicate that these nine events cannot be caused by microlensing. When this analysis is completed, the microlensing sensitivity to high mass objects will reach the vicinity of 1000 solar mass, thus bridging the gap with the recent results on higher mass machos from Yoo et al. (2004), obtained from a study of loosely bound double stars of the galactic halo.

\section{Conclusions}

We have presented the results of the computation of the optical depth for microlensing towards the LMC using a recent picture of the LMC disk. An interesting feature which emerges is a near-far asymmetry of the optical depth for lenses located in the LMC halo, which is not the case for self-lensing. Furthermore, we showed that the timescale distribution of the events and their spatial variation across the LMC disk offers possibilities of identifying the dominant lens population. Through this analysis we have been able to identify a large subset of events that can not be accounted for by self-lensing.

As a general outcome of presently available pixel lensing results, we can clearly infer that the detection of microlensing events towards M31 is now established. The open issue to be still explored is the study of the M31 halo fraction in form of machos. 
Finally, the first account of the analysis of the full EROS2 data set towards the Magellanic Clouds was presented. This data set has presently the largest sensitivity to halo machos. The small number of microlensing candidates is lower than expected from the results of the MACHO group. From this, one derives strict limits on the abundance of machos. A few microlensing candidates have been shown to vary again, many years later; they should be removed from the candidates census.

\section{References}

Afonso, C. et al. 2000, ApJ 532, 340

Afonso, C. et al. 2003, A\&A 400, 951

Alcock, C. et al. 1995, PRL 74, 2867

Alcock, C. et al. 1997a, ApJ 486, 697

Alcock, C. et al. 1997b, ApJ 491, L11

Alcock, C. et al. 2000a, ApJ 542, 281

Alcock, C. et al. 2000b, ApJ 541, 270

Alcock, C. et al. 2001, Nat 414, 617

An, J. et al., 2004, ApJ 601, 845

Aubourg, E. et al. 1993, Nat 365, 623

Baillon, P., Bouquet, A., Giraud-Héraud, Y. \& Kaplan, J. 1993, A\&A 277, 1

Beaulieu, J.Ph. et al. 1995, A\&A 299, 168

Calchi Novati, S. et al. 2003, A\&A 405, 851

Crotts, A.P. 1992, ApJ 399, L43

de Jong, J. et al., A\&A 417, L461

Jetzer, Ph. 1994, A\&A 286, 426

Jetzer, Ph., Mancini, L. \& Scarpetta, G. 2002, A\&A 393, 129

Lasserre, T. et al. 2000, A\&A 355, L39

Mancini, L., Calchi Novati, S., Jetzer, Ph. \& Scarpetta G. 2004, to appear in A\&A and astro-ph/0405257

Milsztajn, A. \& Lasserre, T. 2001, Nucl. Phys. B Proc. Sup. 91, 413

Paczyński, B. 1986, ApJ 304, 1

Palanque-Delabrouille, N. et al. 1998, A\&A 332, 1

Paulin-Henriksson, S. et al. 2003, A\&A 405, 15

Paulin-Henriksson, S. \& Calchi Novati, S. 2004, to appear in the proceedings of the "Rencontres de Moriond 2004': Exploring the Universe"

Renault, C. et al. 1997, A\&A 324, L69

Riffeser, A. et al. 2003, ApJ 599, L17

Tisserand, P. 2004, PhD thesis, University of Nice (in french)

van der Marel, R.P. \& Cioni, M.R. 2001, AJ 122, 1807

van der Marel, R.P. , Alves, D.R., Hardy, E. \& Suntzeff, N.B. 2002, AJ 124, 2639

Yoo, J., Chaname, J. \& Gould, A. 2004, ApJ 601, 311 
This figure "fig04.jpeg" is available in "jpeg" format from: http://arxiv.org/ps/astro-ph/0409496v1 
This figure "fig05.jpeg" is available in "jpeg" format from: http://arxiv.org/ps/astro-ph/0409496v1 